\title{
EDITORIAL
}

\section{STANDARDS OF MEDICAL CARE IN DIABETES-2015- AN EVIDENCED BASED CLINICAL PRACTICE RECOMMENDATION}

The clinical practice recommendation of American Diabetic Association (ADA) renamed their supplemental issue as standards of Medical Care in Diabetes-2015 which contain 14 sections but viewed as a single document. Every section highlighted important topic area with quality evidence and references.

In section 2 the classification of diabetes was highlighted and the changes are important for Asian population as the evidenced for screening in overweight or obese Asian Americans covers a large population in Asians as well. The BMI for screening was changed from $25 \mathrm{~kg} / \mathrm{m}^{2}$ to $23 \mathrm{~kg} / \mathrm{m}^{2}$ as evidenced showed that in Asians the risk of getting diabetes is even more with lower $\mathrm{BMI}^{1,2}$. This also is important for our country as strategies needed to develop for combat the situations and to address the weight gain in different sections of population in the country.

In section 4 , the physical activity for sedentary people were highlighted and evidenced showed that breaking up of sitting $>90 \mathrm{~min}$ at a stretch is important for foundation of diabetic care ${ }^{3}$. The section also describes e-cigarettes are not supported as alternative to smoking to quit smoking ${ }^{3}$.The immunization recommendation was also updated in the supplement giving evidence to provide PCV13 and PPSV 23 vaccination for adults even more than 60 yrs of age beside the routine regular vaccination. The changes are important in our perspective as well as it is cost effective especially for not prolonged sitting and e-cigarettes recommendation. The vaccine although is not cost effective can now be safely advised for senior citizens.

The section 6 of glycamic targets has also been revised and new target is set of $80-130 \mathrm{mg} / \mathrm{dl}$, rather $70-130$ $\mathrm{mg} / \mathrm{dl}$ and the actual average glucose level is more important for reflection than A1C targets ${ }^{4}$. For successful implementation of continuous glucose monitoring (CGM) the ADA recommends on assessing the readiness for $\mathrm{CGM}$ including its support ${ }^{4}$. The flexibility of target eases up in managing diabetes planning and the current CGM hype will also be reconsider carefully by the health care provider.

In section 8, the recommended goal for diastolic blood pressure was changed from $80 \mathrm{~mm} \mathrm{Hg}$ to 90 $\mathrm{mm} \mathrm{Hg}$ for most people with diabetes mellitus and hypertension as evidenced from many RCT showed no extra benefit to reduce DBP below $90 \mathrm{mmHg}$. Regarding statin therapy in Diabetic patients, risk status become more important rather LDL cholesterol level. The screening lipid profile is recommended initial screening for diabetes, at an initial medical evaluation/ or at age 40 years, and periodically thereafter ${ }^{5}$. The evidence for blood pressure target also eased up the health care provider and the patient in their daily living and unnecessary statin therapy will also be curtailed. The risk assessment will be an important tool in many cardiovascular disease recommendation in future. The self oriented screening test will also be reduced through this recommendation as health cost in a country can be influenced as well.

In section 9 of microvascular complications and foot care emphasize to target the high risk for foot complications. Al patients with neuropathy, foot deformity or a history of foot ulcer in diabetic patient should have their feet examined at every visit. The recommendation is important as it is neglected in Bangladesh to look after the care of foot in diabetic patient and frequent examination can easily manage the cases who are prone to develop disability and lack of quality of life. The cost effectiveness of this simple regime is also enormous.

In children and adolescents, new evidence of tight control of glycaemia sowed that A1C of $<7.5 \%$ for all paediatric age groups are sufficient although individualized therapy is still encouraged. The risk and benefit in this regard should be considered by physician. The recommendation will also help our paediatricians to set up goal for their diabetic patients.

In section 12 , the pregnancy and diabetes is a new section that was added to the standards to provide

Bangladesh J Medicine 2015; 26: 47-48 
recommendation related to Diabetes and pregnancy. The highlighting points in this section is preconception counseling, medication, blood glucose targets and monitoring ${ }^{6}$.

The only relevant points of changes are mentioned here in standards of Medical Care in Diabetes-2015 through ADA recommendation. The readers need to complete the reading through different sections of this nice book. The recommendation is simple, evidenced base and practical in much issue. The evidenced are also time demanding and consistent with other guideline of American heart association or relevant organizations. Every twentieth century physician should have adequate knowledge on standards of medical care in diabetes-2015. This documents will help their knowledge, thinking ability and also ease up care in their day to day practice in diabetic patients.

\section{Quazi Tarikul Islam, Md Robed Amin'}

${ }^{1}$ Professor of Medicine, Popular Medical College, ${ }^{2}$ Associate Professor of Medicine, Dhaka Medical College

From the desk of Editor in Chief, BJM

\section{References:}

1. American Diabetes Association. Diagnosis and classification of diabetes mellitus. Diabetes Care 2014;37(Suppl. 1):S81-S90.

2. The International Expert Committee. International Expert Committee report on the role of the A1C assay in the diagnosis of diabetes.Diabetes Care 2009;32:1327-1334.

3. Haas L, Maryniuk M, Beck J, et al. National standards for diabetes self-management education and support. Diabetes Care 2013;37(Suppl.1):S144S153.

4. Miller KM, Beck RW, Bergenstal RM, et al.; T1D Exchange Clinic Network. Evidence of a strong association between frequency of self monitoring of blood glucose and hemoglobin A1c levels in T1D Exchange clinic registry participants. Diabetes Care 2013;36:2009-2014.

5. Goldberg RB, Mellies MJ, Sacks FM, et al.; Care Investigators. Cardiovascular events and their reduction with pravastatin in diabetic and glucoseintolerant myocardial infarction survivors with average cholesterol levels: subgroup analyses in the Cholesterol and Recurrent Events (CARE) trial. Circulation 1998;98:2513-2519.

6. Saudek CD, Herman WH, Sacks DB, Bergenstal RM, Edelman D, Davidson MB. A new look at screening and diagnosing diabetes mellitus. J Clin Endocrinol Metab 2008;93: 2447-2453. 